\title{
La Transpoétique et le normatif chez Hédi Bouraoui
}

\section{Abderrahman Beggar}

URL : http://journals.openedition.org/coma/294

DOI : 10.4000/coma.294

ISSN : 2275-1742

\section{Éditeur}

Institut des textes \& manuscrits modernes (ITEM)

\section{Référence électronique}

Abderrahman Beggar, «La Transpoétique et le normatif chez Hédi Bouraoui », Continents manuscrits [En ligne], 2 | 2014, mis en ligne le 22 avril 2014, consulté le 01 mai 2019. URL : http:// journals.openedition.org/coma/294 ; DOI : 10.4000/coma.294

Ce document a été généré automatiquement le 1 mai 2019.

\section{(c) $(1) \Theta \Theta$}

Continents manuscrits - Génétique des textes littéraires - Afrique, Caraîbe, dispora est mis à disposition selon les termes de la licence Creative Commons Attribution - Pas d'Utilisation Commerciale - Pas de Modification 4.0 International. 


\title{
La Transpoétique et le normatif chez Hédi Bouraoui
}

\author{
Abderrahman Beggar
}

1 L'intérêt porté à Hédi Bouraoui repose sur plus d'une raison. Tout d'abord, il incarne la minorité à plus d'un égard. Né en Tunisie, en 1932, sous l'occupation française, éduqué en France et aux États-Unis, il vit au Canada depuis les années soixante. Son itinéraire est peu typique de l'auteur maghrébin migrant d'expression française, qui s'est souvent installé en France, surtout à Paris ou, du moins, dans une autre métropole francophone en Belgique, en Suisse ou au Québec. Son premier livre, un recueil de poésie intitulé Musoktail (publié aux États-Unis en 1966), porte déjà les signes de la volonté du créateur venu d'ailleurs de passer son message en une langue autre que celle de la société d'accueil. Arrivé au Canada, il choisit Toronto, où il vit toujours. Loin du Québec, il se retrouve en milieu anglophone parmi les membres d'une communauté francophone enclavée. Son œuvre porte ainsi les avatars de la marginalité autant linguistique que culturelle, et de la volonté constamment nourrie de transcender les limitations qu'impose une telle condition. Cette situation explique son attitude critique vis-à-vis de tout centre. Son opposition se base sur sa conscience d'« écrivain migrant qui garde ses identités plurielles. Celles-là font partie de son itinéraire personnel, inscrites dans les gènes qu'il transporte en lui, tel un navire au sillage zigzagant. Ce sont Ces îles qui marchent sans établir de comptoir, sans fonder de royaume. Ainsi, il glisse d'île en île, juste pour recadrer ses écrits dans ses gènes archipéliques. $»^{1}$

2 L'autre raison qui m'amène à m'intéresser à cet auteur réside dans la prolifération de ses écrits et leurs domaines d'exercice : son œuvre couvre poésie, roman, nouvelle, conte et essai, ainsi que des centaines d'articles et de communications lors de conférences internationales. Il arrive aussi à Bouraoui d'écrire en anglais, sans oublier qu'il parle aussi l'arabe, langue de sa Tunisie natale, qui marque son écriture. Son parcours est par ailleurs marqué par sa participation constante aux débats sur la négritude, la francophonie, le surréalisme, le postmodernisme, la mondialisation, le racisme, le dialogue entre les cultures, au point que Albert Memmi l'a qualifié de «chevalier de l'esprit $»^{2}$. Sa pratique de la langue française est marquée par toutes ces influences. 
3 Transpoétique. Éloge du nomadisme, publié en 2005, est particulièrement révélateur de la problématique normative chez Bouraoui. Ce livre qui préconise un nouvel humanisme est constitué de dix-sept chapitres dans un style que l'on peut qualifier d'auto-essai. Si l'autofiction est mise en scène de l'expérience propre sous forme de fiction, dans cet ouvrage, Bouraoui se propose de disséquer l'expérience vécue pour en extraire le matériau de toute une théorie littéraire. Mais, avant de traiter de la question de la norme, il importe d'identifier la nature de celle-ci dans son rapport à l'écriture.

4 Le latin norma vient du grec gnomon, instrument de mesure et d'ajustement. Le terme se réfère aussi au bon usage d'une règle : la parole de l'auteur, considérée comme produit en manque de finition, doit passer sous les fourches caudines d'une instance détentrice du canon. À partir de cette conception, écrire suppose l'assimilation à la norme en partant des lieux d'identification formelle de celle-ci, constitués de traités de lexicologie, de rhétorique, de stylistique, de dictionnaires... Autant de gnomons garantissant la reproduction d'un savoir régulateur qui puise sa puissance dans le respect que lui doit l'expression. Ainsi, le bon usage ne se manifeste-t-il sur le plan du texte littéraire que par bribes. Le mot et la phrase constituent son terrain d'application, et en aucun cas, le texte et ses soubassements en tant qu'objets de création. Celle-ci résulte d'une empreinte spécifique, d'un ensemble de qualités incarnant une subjectivité unique qui échappe au pouvoir normatif. Il ne faut pas oublier non plus que le texte est porteur de l'empreinte d'autres domaines d'exercice du normatif, entre autres l'éthique, le droit, la logique, l'esthétique, pour n'en citer que quelques-uns. Malgré cette pléthore de lieux d'exercice, le savoir que nous avons de la norme reste fragmentaire et ne s'applique qu'à des aspects bien précis. Nous ne pouvons pas parler d'une métascience du normatif, capable de le cerner dans tous ses aspects.

5 Dans le contexte littéraire, la norme (ou les normes) ne peut constituer un modèle, un moule, capable de reproduire l'esprit d'une œuvre. La création ne s'offre pas comme corps organique; ce que l'esprit possédé par l'idée de capter la norme retient, ce sont surtout quelques pièces de la mosaïque, non pas la totalité. Il n'y a pas de recette miracle pour bien écrire. Le rêve d'élaborer une norme totale, capable de nous permettre de créer des textes de qualité, est une pure utopie. Il suffit de regarder comment les nouvelles technologies s'acharnent, sans grand succès, à remplacer la raison et l'imagination humaines, surtout dans le domaine de la traduction, où le respect mécanique de la norme ne permet pas seul d'assurer le passage à l'autre langue. Arrivera-t-on un jour à codifier la norme littéraire dans sa totalité et à créer des ordinateurs capables de la reproduire ? Il est impossible de répondre à une telle question, ce qui n'est d'ailleurs pas le propos de cette étude.

Le siège de la norme reste limité à la conscience de l'écrivain et à celle du lecteur, ce dernier étant représenté par l'éditeur et le critique. Elle suppose l'idée d'adhésion à certaines conventions et sert aussi à créer des hiérarchies et des classifications. La norme est destinée à policer le littéraire et à en garantir les mécanismes de circulation. Elle est aussi ce qui assure un lieu commun, une possibilité de référence collective et, donc, de communication et d'accord sur certaines valeurs esthétiques, morales et autres. Elle ne prend forme que grâce à la pratique. La conscience normative s'identifie à partir de la somme de balises qui permettent de reproduire les mêmes prescriptions. Cette conscience est soumise, entre autres, aux facteurs de l'espace et du temps. Écrire bien ou mal est lié à l'idée d'appartenance et d'exclusion. Le faire s'inscrit dans les limites d'une clôture à plusieurs niveaux: national (par exemple, une écriture française vs 
francophone), générique (à titre d'exemple, les différences entre textes poétiques, théâtraux et journalistiques), circonstanciel (des textes liés à une thématique vendable; par exemple, un événement historique ou un phénomène social), pour ne citer que ces quelques cas.

7 Capter le portrait de la norme telle qu'elle est vécue par le créateur pour constituer ce que nous pouvons traiter comme une symptomatologie de cette interaction : tel est le but de cet article en regard de l'œuvre de Bouraoui. Chaque réaction est reflet du normatif soumis à l'épreuve de la subjectivité du créateur et de ses convictions. Du reste, plutôt que de norme, il serait plus indiqué de parler d'horizon normatif. Le choix de cette orientation part du portrait de la création appliqué à l'art, selon Heidegger, pour qui l'artiste et son œuvre se créent mutuellement. Si l'artiste fait d'un bloc de pierre une sculpture, celle-ci fait de son créateur un sculpteur. En même temps, autant le créateur que son objet adhèrent à un même horizon : l'art ${ }^{3}$. Tout horizon est corps organique dont aucun élément n'est à abstraire: on ne peut pas jouer à déduire un arbre ou une montagne de l'horizon auquel ils appartiennent. C'est cet ensemble qui détermine le cheminement de la création. Un texte littéraire se fait à partir de la conscience éthique, esthétique, légale, rhétorique, idéologique, économique. Tous ces éléments constituent un tout qui ne s'offre pas à la décomposition. Qu'est le propos du travail créatif sinon la soumission de l'horizon à l'épreuve du nouveau qui, à son tour, est susceptible de rejoindre l'usuel ? Toute œuvre est grosse, à un certain degré, de cette même évidence. Cette manière d'agir appelle à mettre le gnomon entre les mains du créateur qui, à ses risques et périls, soumet la langue à ses propres desseins, comme le dit Bouraoui :

J'ai opté pour la langue d'une marâtre qui ne m'aimera jamais comme une mère. Et comme je fais subir à cette langue Vol-taire-Racin-ante les acrobaties les plus inouïes de haute voltige syntactique, sa grammaire ne me pardonnera jamais ${ }^{4}$.

8 Lors d'un entretien privé avec Hédi Bouraoui, la discussion a porté sur le titre plutôt provocateur de son roman, Paris berbère (2011). S'agissait-il d'une satire, d'une manière d'offenser les Parisiens en agitant devant eux l'éventualité qu'un jour la langue de Voltaire céderait devant celle d'Hannibal? De voir ce même Hannibal traverser encore une fois les Pyrénées avec ses éléphants 5 ? L'écrivain cependant donna une autre explication, le titre ayant été imposé par l'éditeur. Bouraoui avait proposé Paris Berbère pour exprimer l'idée du Paris de la Berbère en référence à l'héroïne Tassadite, jeune Kabyle venue étudier à Paris. Selon l'auteur, la raison de ce passage de la majuscule à la minuscule qui changeait radicalement l'idée à l'origine de son projet romanesque réside dans l'orthodoxie de la maison d'édition, son zèle en matière de contrôle $d u$ bon et du mauvais usage de la langue française. Bouraoui a d'abord mené une bataille avant de céder devant la loi des gardiens de la règle.

9 Afin de démontrer la nature de ce genre de censure, l'auteur a accepté de partager avec nous les modifications que l'éditeur lui a demandé d'effectuer dans le manuscrit d'un autre roman, inspiré du Printemps arabe, soumis à l'éditeur en 2012. Ces passages illustrent bien l'exercice du contrôle normatif :

Suggestions de remplacement pour quelques passages à rendre plus neutres (passages pouvant heurter la sensibilité du lectorat et qui pourraient ne pas être publiés).

1) $2^{\mathrm{e}}$ paragraphe à la page 49 :

«Il redit que ce sont les jeunes internautes appuyés par la société civile qui ont réussi à chasser le dictateur et sa clique mafieuse. "

Suggestion de remplacer par: 
"Il redit que ce sont les jeunes internautes appuyés par la société civile qui ont réussi à chasser le dictateur et son groupe de personnes malhonnêtes. "

2) $1^{\mathrm{er}}$ paragraphe à la page 65 :

"Alors le despote a promis d'écraser les opposants, les traitant de rats à tuer et à noyer dans des bains de sang!»

Suggestion de remplacer par:

«Alors le despote a promis d'écraser les opposants, les qualifiant de [...] »

Concernant "rats à tuer et à noyer dans des bains de sang", aucune suggestion ici puisqu'il serait très difficile de réécrire cette phrase pour adoucir le propos tout en conservant le sens. Il faudrait peut-être simplement couper ce passage.

3) Dernier paragraphe à la page 113 et $1^{\mathrm{er}}$ paragraphe à la page 114 :

«Leurs peuples seront des épines empoisonnées dans leurs gueules de margoulins et de futurs despotes! Ils hurleront à tue-tête : « Dégage! Nous n'avons plus peur ! [...] » Suggestion de remplacer par :

«Leurs peuples seront des épines empoisonnées dans leurs figures de petits

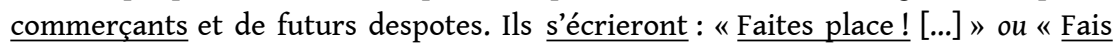
place! Nous n'avons plus peur ! [...]»

4) $4^{\mathrm{e}}$ paragraphe à la page 130 :

«- Oh non! Les Tunisiennes sont plus intelligentes que ces islamistes bornés!»

Suggestion de remplacer par :

«- Oh non! Les Tunisiennes sont plus intelligentes que ces islamistes à l'esprit étroit!" ou «- Oh non! Les Tunisiennes sont plus intelligentes que ces petits islamistes!»

Commentaire :

À la page 77, «goulash » ne s'écrit-il pas «goulasch » avec un c? (Selon le site du CNRTL.)

D'ores et déjà, l'on peut identifier les lieux d'exercice de la norme: le politiquement correct (« passages pouvant heurter la sensibilité du lectorat et qui pourraient ne pas être publiés»), le stylistique (les formes argotiques "gueules de margoulins et de futurs despotes! Ils hurleront à tue-tête: "Dégage!»), le ton («islamistes bornés») et l'orthographe : « goulasch » au lieu de " goulash ». À noter aussi, le rappel de l'autorité du CNRTL (Centre national de ressources textuelles et lexicales). Il est évident qu'au nom du respect de la norme et de la sensibilité du lecteur, bien des qualités du texte sont sacrifiées. Où est passée l'expression de la violence viscérale qui continue à secouer le monde arabe? Ne voit-on pas ici une volonté de domestiquer une parole contestataire peu soucieuse d'accommodation? Couper est le verbe qui exprime le mieux l'impression de Bouraoui quand il conclut notre entretien : «On me prive de ma créativité $»^{6}$, ajoutant : " Tu peux citer tout ce que je t'ai dit, surtout "Je ne veux pas être normal", qui est sorti du fond de mes tripes sans que je m'en aperçoive. » Après de longues négociations, l'éditeur a accepté de garder le verbe dégager parce que l'auteur l'a convaincu qu'un tel mot exprimait « la volonté du peuple tunisien » et que ce vocable fut repris à travers les rues de bien des pays arabes. Ceci montre combien, dans son exercice de l'écriture, cet auteur illustre bien le cas de ceux qui sont aux prises avec les tenants du canon littéraire, euxmêmes se trouvant aussi parfois dans des situations peu enviables, la peur de l'islamisme étant probablement derrière cette position conciliante.

11 La thèse principale de Transpoétique. Éloge du nomadisme est que le monde actuel vit un phénomène d'«éclatement des frontières » et un "bouleversement de l'Histoire causé par les mouvements idéologiques et migratoires $\gg^{7}$; d'où le besoin de traduire ces bouleversements tant sur le plan de la culture que sur celui de l'écriture. La créaculture, néologisme de l'auteur, est l'expression de la nature intrinsèque de l'homme actuel, nourri d'expériences transfrontalières, transculturelles et transnationales, censé jouer un 
rôle actif dans sa propre identité. Sur le plan de la création, de tels bouleversements imposent la nécessité de « réapprendre à entendre, voir, et exprimer autrement le Moi et le Monde $»^{8}$. C'est dans la marche vers un horizon constamment renouvelé, obéissant à la nécessité de reconsidérer l'ancien et d'élaborer de nouvelles manières de dire le monde, que s'inscrit cette esthétique. Ce dynamisme absolu est propre à une vie nomade. Le nomadisme bouraouïen symbolise une liberté absolue qui nie toute frontière (tant physique qu'intellectuelle) et qui encourage l'expérimentation de nouvelles formes d'expression et d'agir, et de critique radicale. Cette manière de considérer le travail créatif repose sur un vitalisme esthétique où les deux ingrédients essentiels sont la surprise et l'élan vital (Bouraoui), jugés «à l'opposé de toute fondation, de toute présence définie. Qu'elle soit géographique ou mentale. " ${ }^{9}$

D'après Bouraoui, la littérature actuelle, considérée surtout dans son centralisme et ses modes de contrôle, doit suivre le rythme effréné que dicte une modernité marquée par les diverses porosités qui menacent les bases traditionnelles, celles de l'appartenance et de l'identité (frontières, cultures, langues, idéologies) comme celles de l'écriture. Pour s'ouvrir sur l'avenir, il faut qu'elle se régénère sachant que « chaque période de l'histoire littéraire possède son "autrement" "

Une telle attitude porte en soi les figures de l'action bouraouïenne vis-à-vis de la norme comme dispositif destiné à limiter son droit à créer sans entraves. En ce sens, il prône une pensée active au service de l'éclatement, qui met en avant l'idée que le faire esthétique doit être déterminé par les signes avant-coureurs d'une fin imminente de l'ordre actuel. Il faut se convertir soi-même en explosif pour traduire l'exigence du changement, faisant de l'acte d'écrire un attentat-suicide comme dans Ignescent (1982), recueil qui met en scène, en cinq actes, une poésie enfantée dans la douleur primordiale, douleur d'accouchement et d'écartèlement : le poète est pris dans un face-à-face virulent avec la vie et la mort. La quatrième de couverture résume le contenu d'une manière fort révélatrice : «Le titre même du recueil Ignescent suggère tout un programme de destruction/construction par le feu de l'amour et du mot, la flamme du verbe et de la vie : igné et naissant [...] » Qualifiées de prosèmes, ces compositions hybrides, où la frontière entre prose et poésie perd toute son étanchéité, livrent une vision contestataire, une invitation à redonner, tant au vécu qu'aux mots qui le traduisent, la force primitive fondamentale qui prélude à l'appropriation par le sens commun. Le regard est attiré par l'œil du cyclone, là où agissent en profondeur les forces vitales. Réduit en miettes, le corps vient compléter ce que la parole n'a pas pu transmettre : le sens absurde de l'existence. Comme pour Camus, l'absurde chez Bouraoui est tout ce qui échappe à l'explication, ces zones broussailleuses et ténébreuses qui résistent encore devant l'assaut de la raison. Le prosème implique un nouveau choix d'expression, une structure aux antipodes de l'usuel et du normatif jugés comme source d'aliénation, ou ce qui empêche le retour à soi. Soi-même n'est plus le centre de l'action. Il faut donc détruire le siège de ce mal considéré comme liberticide. La mort violente du suicidé invite le regard à considérer le corps comme un symptôme de la douleur que suppose la sensibilité aux prises avec l'implacable. Le rejet est porté à son paroxysme quand le suicidé arrive à la conclusion qu'« avec le suicide joyeux, il n'y a plus d'argument $»^{11}$. La parole stérile, qui n'assure plus la liberté d'aller au-delà du constat, devient seuil du néant. Ainsi l'écriture se convertit-elle en activité cathartique qui sert à exorciser la limite.

Bouraoui voit dans les mots le terrain privilégié de ses errances. Si le monde actuel ne permet pas au nomade de vivre selon ses désirs de déplacement dans des étendues sans 
frontières, la page blanche devient le substitut d'une liberté spoliée. Dégager les terrains pour faciliter le mouvement revient à subvertir les modes d'expression et leur linéarité. Ainsi, ses travaux brouillent-ils les registres avec des douzaines de mots peu communs (il préfère les appeler mots-concepts) de la catégorie de l'ignescent, tels que nomadaime, nomadanse, échosmos, iconaison, LivErrance, etc. Que de vocables scindés, hybridés, bricolés pour exprimer l'état de passage et de cheminement des sens ! L'expression perd ainsi son assignation à un même territoire, celui défini par le dictionnaire, pour s'ouvrir sur le pluriel. L'idée de l'amour et celle du nomadisme sortent de leurs lieux respectifs pour rejoindre un autre corps hybride capable d'assumer de nouvelles responsabilités expressives . Cette démarche est enfantée par le vécu. À titre d'exemple, la nomadanse est expliquée par Sammy, le héros de Puglia à bras ouverts, comme la force qui «transporte avec elle toutes les racines qu'elle a acquises sur son chemin ». L'Un représenté par la norme est maintenant soumis au divers. Le nomadanseur accumule au lieu de limiter pour contenir. Le suffixe trans dans transpoétique renvoie à l'idée de transcendance; celle-ci conçue par l'auteur comme embranchement. En conséquence, la destinée du mot n'est pas liée automatiquement à un registre sémantique donné, mais au besoin de mouvance et d'épousailles avec le hasard. L'embranchement donne aux mots une vie autre que celle que leur destine le dictionnaire, et le texte devient un lieu de communion entre des contenus supposés séparés les uns des autres. Ils sont, comme le rhizome deleuzien, une vie qui va vers une autre pour s'accoupler et donner naissance à un être nouveau. La norme subit un effet direct dans ce qui en fait le fondement: à savoir son caractère abstrait. Elle est maintenant appelée non à s'abolir mais à servir une autre cause : celle de la vie. Les mots, selon l'auteur, portent en eux le même souffle qui habite celui qui les prononce; l' embranchement étant "ce qui déclenche une mise en mouvement, en croissance des éléments vitaux de l'arbre de vie des lettres. Il s'agit là donc d'une perpétuelle transcription différenciée qui tend vers la polyvalence transpoétique. $\aleph^{12}$ Cette conception du sens nous rappelle la position de Nietzsche : il n'y a que des forces, et le sens d'un objet dépend de son entrée en contact avec celle avec laquelle il a le plus d' affinités. De ces mots, ce qu'il faut surtout retenir est que le mouvement est endogène : par-delà l'acte de créer, il n'y a que néant. Ici encore, nous pouvons voir la théorie heideggérienne à l'œuvre. Pourtant, il ne faut pas se contenter de cette mise en commun. Que serait l'objet de création si tant le créateur que le créé étaient au service du gnomon? Que serait l'art si la création était soumise au seul règne de l'a priori, et que l'essence déterminait l'existence (ce que Sartre reprochait à la métaphysique)? Devant ces questionnements, il faut aussi s'interroger sur le rôle de l'écrivain, surtout sur le rapport que l'art de nommer entretient avec l'idée de changement, tellement prônée par Bouraoui. Si l'embranchement est "ce qui déclenche une mise en mouvement», il n'est donc plus traité comme l'équivalent d'une boîte magique qui produit intarissablement de nouveaux contenus. Il est tout simplement une force active qui se consume ad infinitum. C'est dans le spectacle de ce feu, cet igne dirait l'auteur, que réside la fonction créatrice. Le premier coup porté à la norme comme ce qui assure continuité et identité immuable du faire littéraire a atteint sa base même. Écrire consiste donc à produire l'énergie capable de nourrir en combustible la forge de la création, le premier concerné étant l'individu actif. Cette énergie, au moment de la mise en mouvement, perd de vue l'horizon et son pouvoir de réduire. Elle remet en question, pour ainsi dire, toute tradition et ouvre son art à la loi de l'expérimental. Cette action obéit à deux mouvements : le premier consiste à arracher le mot à la fixité dictée par le normatif, et le deuxième, à l'ouvrir à ses possibilités 
créatrices. Cette deuxième étape suppose l'entrée en scène de ce que l'auteur appelle béance :

Quand les mots fusent et se fusionnent, échappent aux coups du marteau qui leur auraient fourni leur forme et leur saveur, alors ils s'écoulent dans les béances, ces nappes de disponibilités grandioses, non pour les combler, mais pour les forer... Ne voilà-t-il pas qu'ils en sortent fer forgé de fenêtres ouvertes à toutes les spéculations [...] De la délicieuse Medjerda au majestueux Saint-Laurent, en revenant au Congo et autre Nil cosmogonique, je fluide langue de Seine et de Navarre, en perte de vitesse malgré la vitrine de ses sommets de Chefs d'États francophones. Même si je passe parfois par celle de l'Arno, du Mississippi, du Mécong ou autre Chatt El-Arabs. ${ }^{13}$

Pour s'assurer une souveraineté absolue, le mot a besoin de porter en soi un vide (béance) qui incarne l'illimité du possible, une disponibilité constante à épouser un horizon changeant au rythme des déplacements. Cette béance ignore le préacquiss et assure une ouverture absolue sur le hasard. Elle est propre à la nature des espaces qui s'offrent devant l'inventeur, ceux qui invitent à adopter une attitude ignescente en s'inscrivant dans un "programme de destruction/construction par le feu de l'amour et du mot, la flamme du verbe et de la vie». Ce programme évite l'acte de combler qui dénote ici la correspondance à une définition en usage. La soumission du mot au dictionnaire n'est pas envisageable. Plus encore, c'est la norme qui est attaquée en tant qu'« [o]util, mais aussi [en tant que] modèle de ce qui, en connaissance de cause et selon la droite raison, peut diriger ou orienter une action, une conduite ou un jugement, dans le sens de ce qui doit être jugé » ${ }^{14}$. Au contraire, chez Bouraoui, la destruction/construction cible le modèle et la droite raison pour ne pas se laisser ni diriger ni orienter. D'où le choix du registre aquatique comme fond métaphorique pour l'idée de création: «De la délicieuse Medjerda au majestueux Saint-Laurent, en revenant au Congo et autre Nil cosmogonique, je fluide

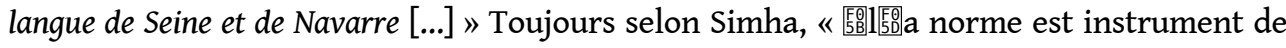
rectitude, mais aussi de mesure universelle permettant d'apprécier ce qui est conforme à la règle et ce qui s'en écarte $\aleph^{15}$. Donc, il faut admettre que toute opposition à la norme rend impossible la lecture. Si la règle grammaticale ou lexicale n'est pas respectée, comment interpréter l'œuvre? En général, l'écriture bouraouïenne est intelligible pour le lecteur, même si quelques-uns de ses poèmes, surtout ceux des débuts, ne sont pas à la portée du lecteur non initié, et ce à cause de leur caractère expérimental, qui invite à l'exploration de domaines de l'expression peu communs. La norme est contestée dans sa propriété de limiter, fixer, clôturer. Le mot ne doit pas être considéré comme ce qui est monumental, érigé à jamais, mais comme une éponge à mille trous (béances) capables d'en assurer la disponibilité devant le dynamisme propre à la vie.

Ces béances ne peuvent servir sans fusionnement, car, pris seul, le mot n'est qu'un jeton dont la valeur dépend de sa circulation ${ }^{16}$. Il lui faut un environnement qui permette d'éviter le marteau, ce sceau du sens commun, pour sortir chargé d'une originalité vitale qui tient au loin les pièges du même. Les porosités propres à la modernité, dont on a parlé ci-dessus, trouvent leur pendant dans l'acte de forer. L'intellectuel n'est pas lié à l'idée de pacte avec l'esprit de son temps. D'où le portrait peu confortable qu'il a de lui-même. En plus de l'acte pénible de forer, en quête de filons et, donc, de nouvelles galeries, il est aussi graveur dans la mesure où il «burine le projet fulgurant d'un avenir possible en transformant de fond en comble tout le langage $»^{17}$. L'opposition à la conformité à la règle passe par un changement de la nature du mot. Celui-ci devient le prolongement de l'errance existentielle de l'auteur. L'homme-fleuve trouve dans le verbe le lieu de concrétisation de ce besoin ontologique. Écrire est nomaditude, relation, communion avec 
le courant qui habite les eaux et connecte avec l'Autre. La transpoétique ne peut s'entendre que si on prend en considération le fond transculturel de l'œuvre. Bouraoui opte pour le transculturalisme comme réponse aux limites du multiculturalisme canadien.

Afin d'échapper à la fixité normative et de réaliser le rêve d'une pensée nomade, les mots fusent. Ce qui assure leur passage inaperçu d'un registre à l'autre. Les écrits de Bouraoui portent les signes de son plurilinguisme. Ses écrits sont parsemés de mots arabes, anglais, espagnols, berbères, créoles et autres. Il part de l'idée que les lieux d'éclosion de sa création (notamment, le Canada, la Tunisie et la France) portent en eux les signes d'une sédimentation culturelle et d'une ouverture qui s'oppose au cloisonnement identitaire et national. Les textes de Bouraoui sont en général traversés, explicitement ou implicitement, de présences linguistiques autres que celle de la langue qu'il choisit pour son écriture. Ce qui explique ainsi son opposition à l'idée d'un amour bilingue de Khatibi qui fait référence au français et à l'arabe Pour Bouraoui, l'écrivain doit renforcer le lien entre les langues qui travaillent en profondeur ses textes; il ne faut pas oublier toutes les langues qui ont marqué de leur empreinte le Maghreb, des Amazighs (ou Berbères) aux Romains, Vandales, Phéniciens, Turcs, Portugais, Espagnols, Français ${ }^{18}$. Il reproche aussi à l'idée de bilinguisme de renfermer la création dans ce qu'il qualifie de binarité infernale. Le Nous vs Eux ne doit pas, selon lui, être l'apanage d'une littérature tournée vers l'humain. Ce sentiment commande ses prises de position vis-à-vis de la langue française, exprimées surtout dans son essai La francophonie à l'estomac (1995), conçu à partir de la perspective de ce qu'il considère comme des francophonies sauvages, à savoir ces enclaves francophones canadiennes dispersées, loin de la Belle Province. Il se positionne ainsi sous le signe d'une opposition au pouvoir centraliste tant de la France que du Québec ${ }^{19}$. Il met l'accent sur tout ce qui est en rapport avec les modes de production et de distribution des œuvres littéraires en français. Sa critique ne s'arrête pas là, mais touche également les francophones ontariens, eux aussi en proie à d'autres modes de segmentation ${ }^{20}$.

Au cours de cette étude, nous avons tenté de démontrer, surtout à partir de Transpoétique. Éloge du nomadisme, comment l'écriture chez Bouraoui est porteuse des symptômes propres à l'expérience de l'auteur, ces symptômes pouvant aller du malaise de la microidentité à l'envie de miner un espace hégémonique et monopolisant. Derrière la volonté de se débarrasser de tout centre se dessine le profil d'une philosophie nomadisante, dirait Bouraoui. À la fixité s'oppose la légèreté d'une volonté créatrice peu soucieuse de s'ancrer ou de s'amarrer à un référent national, ou même identitaire : il faut que l'« unicité [soit] pervertie par la pluralité $»^{21}$. Son attaque contre la francophonie officielle consiste à démontrer comment l'hégémonie du centre est en soi anachronique, surtout à un moment où le transnational, le transculturel, le transfrontalier contestent et évincent peu à peu les définitions usuelles de l'identitaire et du national. Pour Bouraoui, l'écrivain est celui qui négocie constamment la norme et cherche à la pervertir en la soumettant au besoin d'exprimer sa propre vision du monde. 


\section{NOTES}

1. Bouraoui, Hédi, Transpoétique. Éloge du nomadisme, Montréal, Mémoire d'encrier, 2005, p. 10.

2. «Pour Hédi Bouraoui ", Hédi Bouraoui: hommage au poète, textes réunis par Sergio Villani, Woodbridge, Albion Press, 1998, p. 71.

3. Heidegger, Martin, Poetry, language, thought, translated by Albert Hofstadter, New York, Perennial Classics, 2001, p. 17.

4. Bouraoui, Hédi, Transpoétique. Éloge du nomadisme, op. cit., p. 153.

5. L'auteur a consacré une trilogie au personnage d'Hannibal, un immigré tunisien fier de porter le nom de son ancêtre carthaginois, parti en Europe à la recherche de son père : Cap Nord (2008), Les Aléas pour une odyssée (2009) et Méditerranée à voile toute (2010).

6. D'où cette prise de position: «Mais qu'est-ce que la création si elle n'est pas par définition subversion de la norme? Qu'apporte-t-elle sinon une remise en question de sa propre forme pour faire avancer une nouvelle configuration artistique ? Or ce défi inhérent à la littérature dans un contexte de consommation globale est vecteur de toutes sortes de censures. » (Hédi Bouraoui, Transpoétique. Éloge du nomadisme, op. cit., p. 108.)

7. Ibid., p. 50.

8. Ibid., p. 56.

9. Ibid., p. 8.

10. Ibid., p. 56.

11. Hédi Bouraoui, Ignescent. Prosèmes, Paris, Éd. Silex, 1982, p. 15.

12. Hédi Bouraoui, Transpoétique. Éloge du nomadisme, op. cit., p. 43.

13. Ibid., p. 152.

14. Suzanne Simha, « Norme ", Dictionnaire philosophique, sous la direction de Michel Blay, Paris,

Éd. CNRS/Larousse, 2006, p. 567.

15. Ibid., p. 568.

16. Eric Gans, "Originary thinking in a nutshell ", Chronicles of love and resentment $\mathrm{n}^{\circ} 24$, 6 janvier 1996. Accessible à : http://www.anthropoetics.ucla.edu/views/view24.htm

17. Hédi Bouraoui, Transpoétique. Éloge du nomadisme, op. cit., p. 15.

18. En ce sens, la langue sert surtout à assurer la relation, comme disait Glissant à propos du créole : «Ce n'est pas une langue de l'Être, c'est une langue du Relaté. » Édouard Glissant, Le discours antillais, Paris, Gallimard, Folio essais, 2001, p. 141.

19. Les propos suivants sont la preuve de cette révolte: «La francophonie nord-américaine est minée de l'intérieur dans sa production et ses enjeux, parce qu'elle présente un éclatement caractérisé, une dispersion systématique, une résistance à intégrer les difFérentes figures de sa mosaïque littéraire. Le Québec hégémonique et centralisateur occupe avec une certaine arrogance le devant de la scène et ne se soucie que de sa centralité. Ne clame-t-il pas, depuis sa révolution tranquille bien assise, que "hors Québec, point de salut" pour tous les francophones périphériques du pays. » Hédi Bouraoui, Transpoétique. Éloge du nomadisme, op. cit., p. 129.

20. D'où cette description de la manière dont l'ethnoculturel peut constituer l'élément déterminant dans la production littéraire : « En bref, se rangeaient dans la souchique les écrivain (e)s se considérant être les seul(e)s à posséder une appartenance légitime correspondant à une identification authentique et historique à la province [l'Ontario]. Ceux et celles qui sont nés au Canada, doublement minoritaires par rapport à l'anglais et à la majorité francophone québécoise, mais pratiquant la langue officielle de leurs ancêtres nés aussi dans ce pays du dualisme et de l'adversité linguistique. Des enracinés de première instance qui insistent à se distinguer des 
autres écrivain(e)s dits "ethnoculturels" comme si les Souchiques ne possédaient pas d'ethnie.» Hédi Bouraoui, Transpoétique. Éloge du nomadisme, op.cit., p.133. Sur ce point, voir aussi La littérature franco-ontarienne, sous la direction de Hédi Bouraoui, édité par Ali Reguigui, Sudbury, Séries monographiques en sciences humaines, 2000.

21. Hédi Bouraoui, Transpoétique. Éloge du nomadisme, op. cit., p. 45

\section{RÉSUMÉS}

Cette étude de la transpoétique de Hédi Bouraoui traite des positionnements de l'auteur vis-à-vis de la norme littéraire et de son impact sur l'écriture. Nous sommes partis de l'idée d'horizon (selon Heidegger) pour montrer combien la création est porteuse du normatif à plus d'un égard. Le bon ou mauvais usage ne s'inscrit pas seulement sur le plan textuel. Il est symptomatique des rapports, souvent conflictuels, entretenus avec les normes en usage. Ce comportement est aussi lié à la conception du monde qui gère la création.

This paper is about Hédi Bouraoui's transpoetic. The main purpose is to analyze his standpoints towards literary norm and its impact on his writing. Starting out with the concept of horizon according to Heidegger, I proceed to the diverse manifestations of the norm in his creative work. The good or bad use of language is not only taken into account on the textual level. It is symptomatic of the often conflictive nature of the relationship with other normative fields. This is a reality that can't be understood without taking into consideration the world vision behind the creative work.

\section{AUTEUR}

\section{ABDERRAHMAN BEGGAR}

Université de Wilfried Laurier (Waterloo) 